\section{(6) OPEN ACCESS}

\title{
Home enteral nutrition recipients: patient perspectives on training, complications and satisfaction
}

\author{
Karen Boland, ${ }^{1}$ Niamh Maher, ${ }^{2}$ Carmel O'Hanlon, ${ }^{3}$ Maria O'Sullivan, ${ }^{4}$ \\ Niamh Rice, ${ }^{5}$ Martina Smyth, ${ }^{6}$ John V Reynolds ${ }^{3,4,7}$
}

\begin{abstract}
${ }^{1}$ Department of Gastroenterology \& Hepatology, Beaumont Hospital, Dublin 9, Ireland ${ }^{2}$ Community Nutrition and Dietetic Service, Health Service Executive, North County Dublin, Ireland

${ }^{3}$ Department of Nutrition and Dietetics, Beaumont Hospital, Dublin 9, Ireland

${ }^{4}$ Trinity Centre, St James Hospital, Dublin 8, Ireland ${ }^{5}$ NR Consulting, 3 Lumiera, Kilmacanogue, Co., Wicklow, Ireland

${ }^{6}$ Irish Society for Clinical Nutrition and Metabolism, Dublin 18, Ireland

${ }^{7}$ Department of Surgery, St James's Hospital, Dublin 8, Ireland
\end{abstract}

\section{Correspondence to}

Dr Karen Boland, Department of Gastroenterology \& Hepatology, Beaumont Hospital, Beaumont Road, Dublin 9, Ireland; karenjboland@gmail.com

Received 21 July 2016 Revised 12 September 2016 Accepted 18 September 2016 Published Online First 8 October 2016

\section{CrossMark}

To cite: Boland $\mathrm{K}$, Maher $\mathrm{N}$, $\mathrm{O}^{\prime}$ Hanlon $\mathrm{C}$, et al. Frontline Gastroenterology 2017:8:7984.

\section{INTRODUCTION}

The equitable provision of home enteral nutrition (HEN) in the community can have a transformative effect on patient experience and family life for adults and children alike. While optimising quality of life in HEN patients can be challenging, the initiation of HEN positively impacts this measure of healthcare provision. ${ }^{1}$ Quality of life scores have been shown to improve in the weeks after hospital discharge, and HEN is physically well tolerated. However, it may be associated with psychological distress, and sometimes reluctance among HEN patients to leave their homes. ${ }^{2}$ Globally, HEN can attenuate cumulative projected patient care costs through a reduction in hospital admission and complications including hospital acquired infections. ${ }^{3}$ In an era where the cost of disease related malnutrition and associated prolonged hospital stay is being tackled in our healthcare systems, the role of HEN is set to expand. This is a treatment which has clear clinical and social benefits, and may restore some independence to patients and their families. Rather than the indications for HEN being focused on specific diagnoses, the provision of months of quality life at home for patients is adequate justification for its prescription. $^{4}$ Previously, a review of HEN service provision in 39 cases demonstrated that patients want structured follow-up after hospital discharge, and in particular, would like one point of contact for HEN education and discharge..$^{5}$ Management structures, funding challenges and the need for further education, particularly within the primary care setting may limit optimal use of HEN. The Irish Society for Clinical
Nutrition and Metabolism (IrSPEN) aims to develop a national guideline document, drawing on international best practice, forming a template and standards for local policy development in the area of HEN service provision, training and follow-up. The first step in guideline development was to investigate patient experience for adults and children alike. Care needs and supports may differ in these distinct populations. The unmet needs of carers of older adults on HEN have been documented, ${ }^{6}$ although multidisciplinary interventions and evolution of standards for successful discharge will benefit all affected patients and their families. The aim of this study, therefore, was to survey domiciliary HEN clients, to document and analyse user experience, attitudes and complications associated with HEN.

\section{METHODS}

This is a retrospective multicentre qualitative study of patient experience and attitudes relating to HEN and associated support services. In order to evaluate service provision of HEN in Ireland, a self-administered questionnaire was created, to be completed by patients and carers. The final questionnaire was approved after input from hospital and community-based dietitians and patient representatives. Local hospital approval was sought from relevant dietitian managers. Only patients using HEN in the home setting were included. The nutrition and dietetic departments of $17 \mathrm{sec}-$ ondary and tertiary hospitals in Ireland randomly selected patients for completion of this questionnaire with both subjective and objective components. There were no instructions for randomisation 
provided by study coordinators regarding patient selection relating to mode of HEN provision or duration of HEN use. Not all service users completed each section. The results indicate the number and percentages of those who responded. Where a client did not complete a particular question, they were excluded from statistical analysis of that section of service provision and experience. Missing data were excluded in $2 \%(n=2)$ of respondents in analysis of attitudes to HEN.

Data are presented as descriptive statistics. Categorical data were presented as both absolute values and proportions. Two-group comparisons between groups were performed using $\chi^{2}$ analysis and presented using a tabular format. Statistical analysis was conducted using Graphpad, and a p value $<0.05$ denoted statistical significance.

\section{RESULTS}

Eighty-eight clients in 17 hospitals completed this questionnaire with a response rate of $77 \%$. Fifty-two per cent $(n=46)$ of respondents were carers or guardians, 38\% $(n=33)$ were HEN recipients and 10\% $(n=9)$ did not disclose their status. Fifty-seven per cent $(\mathrm{n}=50)$ of survey responders were adults and $57 \%(n=50)$ were male. Forty-two per cent $(n=37)$ of patients had their first feeding tube placed within 2 years of questionnaire completion. The most common indications for HEN in adults were malignancy $(48 \%, n=24)$ and degenerative neuromuscular disorders including Parkinson's disease and multiple sclerosis $(16 \%, n=8)$. Among paediatric respondents, cystic fibrosis, developmental delay and chromosomal or metabolic disorders such as Cri du Chat were the most common underlying pathologies (table 1).

Eighty-nine per cent $(n=78)$ were fed via a gastrostomy tube with $93 \%(n=82)$ of patients using commercially prepared enteral feed. Interestingly, 5\% $(n=4)$ used a mix of commercial formula and blended foods, $1 \%(n=1)$ patient used solely blended food and one patient did not know which feed type was used. Forty-two per cent of respondents used HEN as the sole source of nutrition, and 56\% ( $n=49)$ used this as a supplementary nutrition and were permitted to have an oral diet. The majority of patients included, $83 \%(\mathrm{n}=73)$, use a pump to deliver HEN.

\section{Impact of HEN on daily living}

Eighty-five per cent patients/carers feel confident in tube management and over two-thirds of respondents $(n=60)$ report that management requires little or no effort. Confidence did not relate to the duration of HEN provision. Patients on HEN for $>2$ years were not more likely to feel very confident in management of HEN (91.6\% vs $80 \%$ respectively, $\mathrm{p}=0.27)$.

A quarter of the patients felt that the use of HEN had a significant negative impact on daily activities, but $55 \%(n=48 \%)$ reported little or no impact. In
Table 1 Indications for home enteral nutrition (HEN) use

\begin{tabular}{lc}
\hline & Adults $(\mathrm{n}=50)$ \\
\hline A & \\
Malignancy & $24(48 \%)$ \\
Neuromuscular degenerative disorder & $8(16 \%)$ \\
Stroke & $4(8 \%)$ \\
Respiratory disease & $4(8 \%)$ \\
Brain injury & $3(6 \%)$ \\
Congenital malformation & $3(6 \%)$ \\
Unknown & $4(8 \%)$ \\
\hline & Children $(\mathrm{n}=37)$ \\
\hline B Chromosomal/metabolic disorder & \\
Cerebral palsy & $9(24.3 \%)$ \\
Cystic fibrosis & $7(18.9 \%)$ \\
Developmental delay & $5(13.5 \%)$ \\
Congenital heart disease & $5(13.5 \%)$ \\
Chronic kidney disease & $4(10.8 \%)$ \\
Gastrointestinal tract congenital malformation & $2(5.4 \%)$ \\
Malignancy & $2(5.4 \%)$ \\
Unknown & $1(2.7 \%)$ \\
\hline
\end{tabular}

Patients were asked to complete the indication for initial prescription of HEN as part of the distributed questionnaire. A: Data are displayed as actual and percentage count of adult $(n=50)$ and B: Paediatric $(n=37)$ patients.

children, respondents were more likely to report that HEN impacted on completion of activities of daily living quite a bit, or very much $(\mathrm{p}=0.06)$.

\section{Training provision for HEN}

Hospital nurses were listed as the main source of training for patients and/or carers in $35 \%(n=31)$ of cases (table 2).

Patients did not distinguish between enteral nutrition/gastrostomy clinical nurse specialists (CNS) and ward nurses. Twenty-two per cent $(n=13)$ were trained by the hospital dietitian, and $15 \%(n=13)$ by a nutrition company representative. One patient reported that they received further training with reinforcement and clarification from community services. Twenty-seven per cent $(n=24)$ were unable to identify the profession of their trainer. Most patients felt that training was adequate, with $81 \%(n=71)$ satisfied. A majority of patients were discharged with written information $(86 \%, \mathrm{n}=76)$ and a contact number $(82 \%, \mathrm{n}=72)$ should complications arise (table 2). In addition to formal training, 27\% $(n=24)$ of patients use alternative sources of information to deal with their feeding tubes or troubleshoot complications. These include internet search engines, YouTube videos, a closed Facebook group for parents and parent support groups within the paediatric cohort. 
Table 2 Training and support for home enteral nutrition (HEN) patients after discharge to the community

\begin{tabular}{lccc}
\hline & Adults & Children & $p$ Value \\
\hline Main trainer-hospital dietitian & $16(n=37,43 \%)$ & $2(n=29,7 \%)$ & $0.0017^{*}$ \\
Main trainer-hospital nurse & $13(n=37,35 \%)$ & $20(n=29,69 \%)$ & $0.013^{*}$ \\
Main trainer-company representative & $6(n=37,16 \%)$ & $7(n=29,24 \%)$ & 0.85 \\
Main trainer-other & $2(n=37,5.4 \%)$ & $0(n=29,0 \%)$ & 0.22 \\
Written info given on discharge & $45(n=50,90 \%)$ & $31(n=34,91 \%)$ & 0.87 \\
Support-community dietitian & $9(n=50,18 \%)$ & $8(n=36,22 \%)$ & 0.45 \\
Support-general practitioner & $15,(n=50,30 \%)$ & $4(n=36,11 \%)$ & $0.037^{*}$ \\
Carer assistance for HEN administration & $4,(n=50,8 \%)$ & $9(n=37,24 \%)$ & $0.039^{*}$ \\
\hline
\end{tabular}

Differences in HEN support, training and information were identified between adults and children. Data represent actual number and respondents within each section shown $(\mathrm{n})$. In total, 88 respondents were included, but those who were not aware of their trainer's title, or who omitted sections pertaining to this table were excluded from data analysis. Statistical significance determined after analysis with Fisher exact test and $\chi^{2}$ tables.

${ }^{*}$ Denotes statistical significance with $p<0.05$.

\section{Support for complications associated with HEN after discharge}

Many patients require externally sourced assistance such as funded carers/nurses or non-immediate family members to manage their feeding tube and HEN. While almost half of the respondents coped without any assistance, $36 \%(n=32)$ avail of family support and $15 \%(n=13)$ need help from nurses and carers. In most cases, professional support for HEN and feeding-tube complications is provided by hospitalbased healthcare workers. The primary source of support and monitoring in over one-third of cases $(n=31)$ is the hospital dietitian, and the enteral nutrition/gastrostomy CNS in a quarter of patients $(n=22)$. Training provided by a CNS occurs in the paediatric cohort as there is a lack of adult CNS managing enteral nutrition in Ireland. Overall, community-based care is limited, with support from the community dietitian in only $8 \%(n=7)$, the public health nurse in $10 \%(n=9)$, the nutrition and feeding tube companies in $15 \%(n=13)$, community pharmacist $(7 \%, n=6)$ and their general practitioner in only $2 \%(n=2)$ cases (table 2). Almost half of those surveyed felt that there were very good or excellent supports available, but $20 \%(n=18)$ experienced fair and 7\% $(n=6)$ reported poor support.

\section{Differences in training and support between adults and children}

We performed a sub analysis of adult and paediatric service users to identify variables in patient experience, given that clinical nutrition services are provided in a different manner in these groups. In the paediatric population, patients/carers were three times more likely to have support from state provided carers for day-to-day HEN provision $(p=0.039)$ (table 2$)$. This may reflect the complex care needs underlying the indication for HEN in the paediatric population. We also found statistically significant differences in training and community support between adult and paediatric populations. The primary training source for HEN is more likely to come from the hospital dietitian for adults $(\mathrm{p}=0.0017)$, and hospital-based nursing staff for children and their families $(p=0.013)$ (table 2). A minority of both adults and children are trained predominantly by nutrition company representatives.

Over $90 \%$ of patients received written instructions on the management of HEN in both groups.

After discharge, there were variable reports of community-based supports for ongoing care. Only $18 \%$ adults and $22 \%$ of children were supported by a community-based dietitian and less than 50\% recall support for feeding-tube and HEN provision from their local public health nurse. While this was common to both adults and paediatric patients, adults were significantly more likely to receive assistance or support in HEN management from their general practitioner $(30 \%$ in adults, $18 \%$ in children, $\mathrm{p}=0.037)$ (table 2). However, less than a third of adults received any assistance in HEN management from their general practitioner.

\section{Complications associated with HEN}

Feeding tube complications and adverse effects were broadly similar in both groups. We evaluated HEN-related complications (table 3), with the highest incidence of complications identified as feeding tube blockages $(30 \%, n=26)$, infections $(48 \%, n=42)$ and vomiting $(27 \%, n=24)$. Vomiting was significantly more likely to occur as a complication in the paediatric cohort $(p<0.0001)$. There were slightly increased reports of stoma site infection in children $(p=0.083)$ (table 3).

The majority of these complications can be successfully managed in the community, reducing tertiary healthcare use and rates of admission for these indications. This will be assisted by increasing postdischarge supports and alternative healthcare access points, hence improving patient experience and outcomes.

Almost a quarter of patients have experienced sudden dislodgement of their feeding tube. Approximately half of the respondents required 
Table 3 Complications associated with home enteral nutrition (HEN)

\begin{tabular}{|c|c|c|c|c|}
\hline & Total $(n=87)$ & Adults $(n=50)$ & Children ( $n=37$ ) & $\mathrm{p}$ Value \\
\hline Feeding tube replacement & $40(46 \%)$ & $21(42 \%)$ & $19(51 \%)$ & $0.04^{*}$ \\
\hline Tube replacement in $A \& E$ department & $14 / 40(35 \%)$ & $10(48 \%)$ & $4(24 \%)$ & $0.039 *$ \\
\hline Pump malfunction & $2(2 \%)$ & 0 & $2(5 \%)$ & 0.096 \\
\hline Tube dislodgement & $21(24 \%)$ & $12(24 \%)$ & $9(24 \%)$ & 0.9 \\
\hline Broken feeding tube & $9(10 \%)$ & $5(10 \%)$ & $2(11 \%)$ & 0.9 \\
\hline Stoma site infection & $40(46 \%)$ & $19(38 \%)$ & $21(57 \%)$ & 0.083 \\
\hline Blocked feeding tube & $26(30 \%)$ & $15(30 \%)$ & $11(30 \%)$ & 0.98 \\
\hline Vomiting & $24(27.5 \%)$ & $5(10 \%)$ & $19(51 \%)$ & $<0.001^{*}$ \\
\hline Diarrhoea & $16(18 \%)$ & $8(16 \%)$ & $8(22 \%)$ & 0.5 \\
\hline Constipation & $23(26 \%)$ & $11(22 \%)$ & $12(32.4 \%)$ & 0.27 \\
\hline Weight loss & $10(11.5 \%)$ & $7(14 \%)$ & $3(8 \%)$ & 0.39 \\
\hline
\end{tabular}

Patients and their carers were asked to outline complications associated with HEN including side effects associated with feeding and technical difficulties including pump dysfunction and feeding tube issues. Data show total percentages and numbers, and are further subdivided into adult and paediatric patients. Data were analysed using Fishers exact test and $\chi^{2}$ tests to identify differences in the experience of adult and paediatric patients.

*Denotes statistical significance, determined by $p<0.05$.

feeding tube replacement since discharge to the community. Interestingly, while there were many similarities, we noted that children were less likely to attend the emergency department for feeding tube replacement than adults $(\mathrm{n}=4$ and $\mathrm{n}=10$, respectively; $\mathrm{p}=0.079$ (table 3)). Most replacements for children occurred in the outpatient department and in some cases for adults, through direct admission to the endoscopy department. Three adults $(\mathrm{n}=19)$ and four children $(n=21)$ had tube replacements in their home environment; this is likely to reflect elective feeding tube replacement. Both adults and children were more likely to have had tube replacement if they have had HEN for $>2$ years $(p=0.0048$ and $p<0.0001$, respectively (table 3 )).

\section{DISCUSSION}

Based on the volume of tube feeds supplied, and the number of pumps serviced and provided in 2013, an estimated 2500 patients use tube feeding in Ireland. Approximately 1900 of these are community-based patients. We anticipate that as demand for home enteral feeding has increased, the number of patients is also likely to have increased to greater than 2000 patients (Niamh Maher and Niamh Rice, Personal Communication 10 July 2016). Without a prospectively maintained national database, we have no accurate number of patients discharged on HEN annually. A service evaluation by one regional community dietetics service in 2015 reported, on average, 39 new referrals annually to their service since 2008 , increasing year on year. ${ }^{7}$ Focused efforts are required to improve the structures currently in place, and to optimise care in the community for patients receiving HEN.

The survey serves to inform us of gaps in HEN services and will be used to ensure that our standards and guidelines will meet patient needs in both adult and paediatric populations. It voices the positive experience of patients dealing with the HEN Dietitian in facilitating discharge to home. The remarkable ability of adult HEN patients to cope with complications and challenges has been documented previously, ${ }^{8}$ although with inconsistent and often infrequent follow-up after discharge, ${ }^{9}$ and low patient satisfaction with post-discharge supports. ${ }^{10}$ A number of research projects have studied patient experience and effect on quality of life. Families and patients report significant stress which may be linked to complications of HEN. Overall, patients seem to quickly gain competence in management of their HEN in the face of a marked life-changing event. While this questionnaire was not designed to formally assess the impact of HEN on quality of life, the confidence of HEN users is reassuring, and three-quarters of respondents do not report significant limitation of activities of daily living as a direct result of HEN. The majority of patients in this study used feeding pumps for HEN administration $(83 \%, \mathrm{n}=73)$. Future analysis should aim to enrol more patients using gravity bag feeding to analyse differences in experience in comparison with pump feeding.

The relative safety of feeding tube insertion, the ability to place these safely in patients who may have significant co-morbidities, combined with improved quality of life and an impetus to transfer patient care to the community for economic reasons have come together to steadily increase the number of patients discharged on HEN. ${ }^{11}$ We believe that there should be a single point-of-contact for clients and their families to ensure consistent and complete training processes nationally, and comprehensive discharge planning. The point-of-contact would also train service users in how to access reliable information expediently when trouble-shooting pump and HEN complications. Poor quality planning for discharge to 
the community and a lack of consistent support at home contribute significantly to negative beliefs and experiences of HEN use. ${ }^{6} 912$

This questionnaire also highlights the need to involve primary care and community-based healthcare professionals in HEN management and follow-up, requiring financial resourcing and development of educational programmes. Previously, general practitioners have reported a lack of knowledge, poor communication between primary and secondary care and an unsatisfactory experience when dealing with HEN. ${ }^{13}$ Patients and their families have reported poor support from primary care providers. $^{8}{ }^{9}$ Feeding-tube-related complications result in increased healthcare utilisation, although many of these complications may be successfully managed in the community with appropriate training. ${ }^{14}$ This is an aspect of HEN service provision which will require planning and outreach to these healthcare workers.

Internationally, there is a renewed emphasis and a spotlight on nutrition as a cornerstone of care in the development of health policy. ${ }^{15}$ Previously, differences in the indications for long-term HEN and variances in terms of administration and funding models have been outlined in Europe. ${ }^{16}$ Clearly there is a role for streamlined and standardised management of these patients within distinct healthcare models. Focusing on provision of evidence-based patient advice aiming

\section{Significance of this study}

\section{What is Known?}

- Home Enteral Nutrition (HEN) improves quality of life and offers financial savings, facilitating discharge to the community and reducing the risk of healthcarerelated infections.

- HEN may be associated with psychological distress as patients and carers adjust to this life-changing treatment.

What this article adds?

- Patient experience of HEN is largely positive, but key areas for improvement in the process of discharge planning have been identified as this patient population expands.

- This study supports the need for additional training of existing primary care providers in the management of complications associated with HEN.

\section{How might it impact on clinical practice in the}

foreseeable future?

- A single point-of-contact after discharge may improve patient experience and also reduce avoidable hospital admissions, particularly for gastrostomy replacement.

- This questionnaire will be used to develop national standards for HEN services and community monitoring. to consolidate patient confidence and streamlined pathways for monitoring and feeding-tube replacement may limit unnecessary inpatient hospital stays with clear financial and social benefits. We propose that the introduction of imminent guidelines for the management of this patient group will improve the experience of trainers, healthcare workers and HEN patients, and will support current momentum to improve their nutritional status.

Acknowledgements We gratefully acknowledge the assistance of local hospital and community dietitians in all associated hospitals in development and distribution of study materials. We also acknowledge the support and assistance of the Hospital Dietitian Managers Group and members of the IrSPEN Policy and Practice and Management Committees.

Contributors $\mathrm{KB}$ analysed the questionnaire results, composed the manuscript and performed statistical analysis on the data. $\mathrm{NM}, \mathrm{COH}, \mathrm{MOS}$ and NR contributed to development of the study questionnaire and the composition of the manuscript and revisions. MS coordinated questionnaire distribution and patient participation. JVR contributed to concept development and manuscript composition.

Competing interests None declared.

Provenance and peer review Not commissioned; externally peer reviewed.

Open Access This is an Open Access article distributed in accordance with the Creative Commons Attribution Non Commercial (CC BY-NC 4.0) license, which permits others to distribute, remix, adapt, build upon this work noncommercially, and license their derivative works on different terms, provided the original work is properly cited and the use is non-commercial. See: http://creativecommons.org/licenses/by$\mathrm{nc} / 4.0 /$

\section{REFERENCES}

1 Schneider SM, Pouget I, Staccini P, et al. Quality of life in long-term home enteral nutrition patients. Clin Nutr 19:23-8.

2 Roberge C, Tran M, Massoud C, et al. Quality of life and home enteral tube feeding: a French prospective study in patients with head and neck or oesophageal cancer. Br J Cancer 1999;82:263-9.

3 Ojo O. The challenges of home enteral tube feeding: a global perspective. Nutrients 2015;7:2524.

4 Howard L, Ament M, Richard Fleming C, et al. Current use and clinical outcome of home parenteral and enteral nutrition therapies in the United States. Gastroenterology 1995;109:355-65.

5 Shortall C, Aherne M, Boland S, et al. Hospital to home paediatric enteral nutrition-parents need support. Irish Med J 2015;108:46-8.

6 Silver HJ, Wellman NS, Galindo-Ciocon D, et al. Family caregivers of older adults on home enteral nutrition have multiple unmet task-related training needs and low overall preparedness for caregiving. J Am Diet Assoc 2004; 104:43-50.

7 Maher N, Gerlitz H. Home Enteral Nutrition Service in North Dublin. IrSPEN Conference 2015: Health Service Executive, 2015.

8 Halliday V, Baker M, Thomas AL, et al. Patient and family caregivers' experiences of living with a Jejunostomy feeding tube after surgery for esophagogastric cancer. JPEN J Parenter Enteral Nutr 2015. 
9 McNamara EP, Flood P, Kennedy NP. Enteral tube feeding in the community: survey of adult patients discharged from a Dublin hospital. Clin Nutr 2000;19:15-22.

10 L'Estrange F. An audit of adult patients on home enteral tube feeding in a region of Northern Ireland. J Hum Nutr Diet 1997;10:277-87.

11 Elia M. An international perspective on artificial nutritional support in the community. Lancet 345:1345-9.

12 Bjuresäter K, Larsson M, Athlin E. Patients' experiences of home enteral tube feeding (HETF)—a qualitative study. J Res Nurs 2015;20:552-65.
13 Madigan SM, Fleming P, McCann S, et al. General Practitioners involvement in enteral tube feeding at home: a qualitative study. BMC Fam Pract 2007;8:29-29.

14 Crosby J, Duerksen D. A retrospective survey of tube-related complications in patients receiving long-term home enteral nutrition. Dig Dis Sci 2005;50:1712-17.

15 Authority HIaQ. National Standards for Safer Better Healthcare. Health Service Executive, 2012.

16 Hebuterne X, Bozzetti F, Moreno Villares JM, et al. Home enteral nutrition in adults: a European multicentre survey. Clin Nutr 22:261-66. 